\title{
When Stream Cipher Analysis Meets Public-Key Cryptography
}

\author{
Matthieu Finiasz and Serge Vaudenay \\ EPFL \\ CH-1015 Lausanne - Switzerland \\ http://lasecwww.epfl.ch/
}

\begin{abstract}
Inspired by fast correlation attacks on stream ciphers, we present a stream cipher-like construction for a public-key cryptosystem whose security relies on two problems: finding a low-weight multiple of a given polynomial and a Hidden Correlation problem. We obtain a weakly secure public-key cryptosystem we call TCHo (as for Trapdoor Cipher, Hardware Oriented). Using the Fujisaki-Okamoto construction, we can build an hybrid cryptosystem, $\mathrm{TCHo}^{n}$-FO, resistant against adaptive chosen ciphertext attacks.
\end{abstract}

\section{Introduction}

Many of nowadays cryptosystems rely on the problem of factoring numbers. With the RSA 24] cryptosystem, the key recovery problem is equivalent to factoring a modulus into prime numbers. The message recovery problem is an ad-hoc problem, assumed to be hard, but potentially easier than factoring. To setup the cryptosystem we first generate secret prime numbers and then multiply them together to get the public key. Although the hardness of the factoring problem is an important open problem, it is known to be easy by using quantum computers [26.

With polynomials, the factoring problem is essentially easy. However, the problem of finding a multiple with low degree and weight is presumably hard. This problem occurs in correlation attacks on stream ciphers, and no polynomial time algorithm exists to solve it. Therefore, we can setup a new trapdoor system by first generating a secret low-weight polynomial and then looking for a suitable factor to produce a public key. We thus derive a new cryptosystem consisting simply of the XOR of two LFSRs with a random noise source. One LFSR is used to encode the data, the other contains the trapdoor. It is only used to hide the data, and the noise source provides non-linearity. The ciphertext is the result of this XOR. The key recovery problem is equivalent to finding a low-weight multiple of a given polynomial. The message recovery problem is an ad-hoc problem, potentially easier but still (assumed to be) hard: the Hidden Correlation (HC) problem. It further may remain a hard problem with quantum computers.

We think that analyzing this cryptosystem would be important in any case because this would either provide us with a secure post-quantum cryptosystem 
(if not an efficient pre-quantum one), or with new improvements for existing correlation attacks against stream ciphers. We aim at making the first step in this direction.

In this paper we review existing algorithms for finding low-weight multiples of a given polynomial and for the decoding of a noisy LFSR. This provides heuristics to select parameters for TCHo, a one-way public-key encryption scheme. It can encrypt small blocks of up to 30 bits into ciphertexts of a few thousand bits. $\mathrm{TCHo}^{n}$ encrypts $n$ such blocks independently. The stream-cipher-like structure of our construction makes it most suitable for hardware implementation: an ASIC of a few thousand gates (equipped with a randomness generator) at $4 \mathrm{MHz}$ should be able to encrypt a block in less than $4 \mathrm{~ms}$.

We believe TCHo could also fit on a passive RFID tag. Providing public-key encryption to RFID tags leads to new opportunities to solve privacy/security issues in RFID protocols. Current protocols with readers connected to a single server 123] are based on symmetric cryptography and either compromise privacy or induce an important overhead complexity on the reader side. Public-key cryptography is the simplest (and most reliable) solution to solve these issues, however, no public-key encryption primitive can fit on an RFID tag. TCHo could possibly be the first construction to achieve this.

We have implemented the TCHo construction in $\mathrm{C}++$ using NTL [27]. Key generation is pretty slow (about 4 minutes) as it requires to factorize polynomials with huge degrees. Encryption takes a fraction of a second in software, but this time is not significant for hardware implementation: LFSRs are not well suited for use with a CPU. Finally, our decryption implementation takes a few seconds.

Previous work. Quite a lot of work has been done trying to attack various stream ciphers like constructions. The first attacks were the (fast) correlation attack, invented by Siegenthaler [28] and improved by Meier and Staffelbach [20]. The main idea is to isolate one linear feedback shift-registers (LFSR) inside a stream cipher and approximate the rest of the construction by another large LFSR with some noise, then try to recover the initialization of the first LFSR and get a part of the key. Many improvements and applications to specific constructions have been made 451314151821. This work was inspired by a preliminary attack on Bluetooth E0 17,19. Some of the most efficient techniques are compared in Section 2.2.

A key problem in most of these techniques is the possibility to find low-weight multiples of a given polynomial. The best techniques rely either on exhaustive search or on decoding techniques $342 / 2230$. Section 2.1 of this article is devoted to this problem.

More recently, algebraic attacks have been applied to break some constructions 6/781011]. These attack can be very efficient but, even if some bounds have been proven [79], predicting their efficiency is quite a difficult problem. Throughout this article we will neglect this category of attacks as they are not well suited for large randomized constructions like ours.

The concept of trapdoor in a stream cipher was already brought up by Camion, Mihaljevic and Imai [2]. They showed that using specific retroaction polynomials 
in LFSRs, some bits of the output stream could depend on only some bits of the initialization, making it possible to speed up the recovery process for constructions which otherwise seemed perfectly sound. This can be well suited to kleptographic attack but it seems hard to build a public-key cryptosystem this way.

In Section 2, we define new computational problems and provide heuristics to solve them. This suggests some parameters ranges in which they can be reasonably assumed to be hard on average. Then we define a raw encryption scheme in Section 3 and prove that it is one-way under chosen plaintext attacks.

\section{New Computational Problems}

In this section, we formalize some computational problems which will be used and review existing algorithms to solve them. We focus on exact complexities as opposed to asymptotic ones. For this, we use two constants $c_{\text {easy }}$ and $c_{\text {hard }}$ to indicate that a complexity below $2^{c_{\text {easy }}}$ operations is essentially easy in practice and efficient, and that a complexity over $2^{c_{\text {hard }}}$ is intractable in practice. In practice, we can take $c_{\text {easy }}=30$ and $c_{\text {hard }}=80$.

Throughout this paper, we will define the bias of a source as bias $(\mathcal{S})=P(\mathcal{S}=$ $0)-P(\mathcal{S}=1)$. This bias is hence taken between -1 and 1 and is null when the source is unbiased. In our system, we will only consider positive biases, that is, sources which produce more 0's than 1's.

\subsection{The Low-Weight Polynomial Multiple Problem}

As opposed to integers, factoring polynomials is essentially easy. But when it comes to finding low-weight polynomial multiples, the problem becomes harder (at least, it is not known to be easy). Here, we will only focus on polynomials over $\mathbf{F}_{2}$.

Problem 1 (Low-Weight Polynomial Multiple). We consider the following problem on average over the random selection of an instance $P$, issued by a given instance generator Gen. We say that the problem is hard for Gen if solving it requires more than $2^{\text {chard }}$ complexity on average. We consider two kinds of generator. Let $\mathrm{Gen}_{1}$ be a generator which selects a random primitive polynomial $P$ of degree $d_{P}$. Let $\mathrm{Gen}_{2}$ be a generator which selects a random polynomial $K$ of degree $d_{K}$ and weight $w$ until it has a primitive factor $P$, produced as the output, whose degree $d_{P}$ is in a given interval $\left[d_{\min }, d_{\max }\right]$.

Parameters: a weight $w$, two degrees $d_{P}<d_{K}$

Instance: a binary polynomial $P$ of degree $d_{P}$

Problem: find a multiple of $P$ with degree at most $d_{K}$ and weight at most $w$

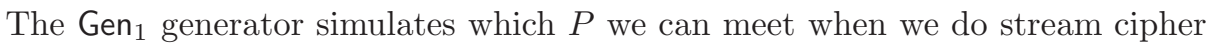
cryptanalysis. The $\mathrm{Gen}_{2}$ generator simulates which $P$ we can meet when we construct a public-key cryptosystem. 
Note that if $P$ is irreducible, if $x$ has order $n \leq d_{K}$ in the group $\left(\mathbf{F}_{2}[x] / P(x)\right)^{*}$, and if $w \geq 2$, then $K=x^{n}+1$ solves the problem. Taking $P$ primitive ensures that the order $n=2^{d_{P}}-1$ is maximal. Also, for values of $w$ greater than the Hamming weight of $P$, then $P$ itself solves the problem. However, for $d_{K}$ and $w$ small, the problem is believed to be hard. There are several strategies to solve it. Heuristically, if $P$ is generated by $\mathrm{Gen}_{1}$, the average number of solutions with nonzero constant term is

$$
\mathcal{N}_{\mathrm{sol}}=2^{-d_{P}} \sum_{i=0}^{w-1}\left(\begin{array}{c}
d_{K} \\
i
\end{array}\right) \approx 2^{-d_{P}}\left(\begin{array}{c}
d_{K} \\
w-1
\end{array}\right) .
$$

When using $\mathrm{Gen}_{2}$, the average number of solutions becomes $1+\mathcal{N}_{\text {sol }}$.

Strategy 1 - The birthday paradox. This strategy consists in building two lists of polynomials which are sums respectively of 0 or 1 and of a polynomial with weight $\frac{w-1}{2}$ and null constant term, all reduced modulo $P$. Once this is done, one simply looks for collisions. The lists have a size of $L=\left(\begin{array}{c}d_{K} \\ (w-1) / 2\end{array}\right)$ and the complexity is $\mathcal{O}\left(L\left(\log (L)+d_{P}\right)\right)$. This strategy is always faster than exhaustive search, but requires a lot of memory.

Strategy 2 - Wagner's generalized birthday paradox. When the number of solutions becomes large enough (of order $\mathcal{O}\left(2^{d_{P} / 3}\right)$ ), techniques based on Wagner's generalized birthday paradox 30] can become more efficient. This algorithm is not fit for finding all possible polynomials (or the hidden one from $\mathrm{Gen}_{2}$ ) but can find one solution among many. If there exists $a \geq 2$ such that $\left(\begin{array}{c}d_{K} \\ (w-1) / 2^{a}\end{array}\right) \geq 2^{d_{P} /(a+1)}$, then one solution can be found with a complexity $\mathcal{O}\left(2^{a} 2^{d_{P} /(a+1)}\right)$.

For instance, when $d_{K} \geq 2^{d_{P} /\left(1+\log _{2}(w-1)\right)}$, we can use $a=\log _{2}(w-1)$ and find a multiple within $\mathcal{O}\left((w-1) d_{K}\right)$. Clearly, such a low complexity cannot be reached for any $w \leq d_{P}$ when $d_{K} \leq 2^{d_{P} /\left(1+\log _{2}\left(d_{P}-1\right)\right)}$.

Strategy 3 - Syndrome decoding. Solving the problem can be done using a syndrome decoding algorithm. Compute the matrix of all the $x^{i} \bmod P(x)$ for $i$ from 1 to $d_{K}$ and then find a low-weight word in the preimages of 1 of this matrix. When a single solution exists, this has a cost of:

$$
\mathcal{O}\left(\mathcal{P} \text { oly }\left(d_{K}\right)\left(\frac{d_{K}}{d_{P}}\right)^{w-1}\right),
$$

where $\mathcal{P}$ oly $\left(d_{K}\right)$ is a polynomial of degree 2 or 3 in $d_{K}$ (see [16] for example). We neglect this polynomial part as improved algorithm like [3] can compensate it. This complexity holds when there is a unique multiple polynomial of degree $d_{K}$ and weight $\leq w$. When there are more solutions this cost is approximately divided by $\mathcal{N}_{\text {sol }}$.

Strategy 4 - Exhaustive search. When looking for multiples of degree just above $d_{P}$, an exhaustive search on $Q$ such that $K=P \times Q$ can be faster. The complexity of finding all multiples is $\mathcal{O}\left(\mathcal{P}\right.$ oly $\left.\left(d_{K}\right) 2^{d_{K}-d_{P}}\right)$. 
The best algorithm for finding low-weight multiples depends on both the parameters and our objective (whether we want to find a single, many, or all solutions). When a single solution exists, the best choice is Strategy 3. This leads us to the following assumption

Assumption 2 (Low-Weight Polynomial Multiple). When $w \log _{2} \frac{d_{K}}{d_{P}} \geq$ $c_{\text {hard }}$ and $\left(\begin{array}{c}d_{K} \\ w-1\end{array}\right) \leq 2^{d_{P}}$, the low-weight polynomial multiple problem is hard on average for $\mathrm{Gen}_{1}$. When $w \log _{2} \frac{d_{K}}{d_{\min }} \geq c_{\text {hard }}$ and $\left(\begin{array}{c}d_{K} \\ w-1\end{array}\right) \leq 2^{d_{\min }}$, the low-weight polynomial multiple problem is hard on average for $\mathrm{Gen}_{2}$.

\subsection{The Noisy LFSR Decoding Problem}

A binary linear code of length $\ell$ is a vector subspace of $\{0,1\}^{\ell}$. Elements are codewords. We consider the problem of decoding noisy strings, i.e. decoding the XOR of a codeword together with the output of a random source $\mathcal{S}_{\gamma}$ with bias $\gamma$. This source represents the error produced by a binary symmetric channel. In what follows we concentrate on codes which consist of all possible $\ell$-bit strings which can be output from an LFSR $\mathcal{L}_{P}$ with a fixed retroaction polynomial $P$ of degree $d_{P}$, i.e. sequences $Z=\left(z_{1}, \ldots, z_{\ell}\right)$ such that $\left(z_{t}, \ldots, z_{t+d_{P}}\right) \cdot P=0$ for $t=1, \ldots, \ell-d_{P}$ (where $\cdot$ denotes a scalar product, which means we consider $P$ as a binary $\left(d_{P}+1\right)$-tuple). We formalize the decoding problem of the noisy LFSR channel as follows.

Problem 3 (Noisy LFSR Decoding). We consider the following problem on average over the random selection of $P, X$, and the biased noise. We say that the problem is hard if getting a single bit of $X$ (that is, decoding $X$ with probability higher than $2^{1-d_{P}}$ ) requires over $2^{c_{\text {hard }}}$ complexity on average.

Parameters: a length $\ell$, a polynomial $P$ of degree $d_{P}$, a bias $\gamma$

Noisy LFSR channel: given a uniformly distributed random seed $X$ of length $d_{P}$, generate $Y$, the XOR of the output of length $\ell$ of $\mathcal{L}_{P}$ initialized with $X$ and a random noise generated by $\mathcal{S}_{\gamma}$

Problem: given $Y$, recover $X$

When $\gamma$ is so close to 1 that errors are unlikely (e.g. $1-\gamma \ll \ell^{-1}$ ), the problem can easily be solved with high probability of success by Gaussian elimination. When $\gamma$ is so small that we cannot even distinguish $\mathcal{S}_{\gamma}$ from an unbiased source (e.g. $\gamma \ll \ell^{-\frac{1}{2}}$ ), the problem is impossible to solve with relevant probability of success. In what follows we may assume that the channel transmits less data than its capacity $1(\gamma)=1+\left(\frac{1}{2}+\frac{\gamma}{2}\right) \log _{2}\left(\frac{1}{2}+\frac{\gamma}{2}\right)+\left(\frac{1}{2}-\frac{\gamma}{2}\right) \log _{2}\left(\frac{1}{2}-\frac{\gamma}{2}\right)$, i.e. $\frac{d_{P}}{\ell} \leq C(\gamma)$. Thus, with unbounded computational power, decoding is possible. However, this is not always the case in only $\mathcal{O}\left(2^{c_{\text {hard }}}\right)$ operations.

Three main classes of algorithms exist to solve this problem: those based on information set decoding, those trying to perform maximum likelihood (noted

${ }^{1}$ When $\gamma$ is small, we have $C(\gamma) \approx \frac{\gamma^{2}}{2 \log 2}$ (with less than $1 \%$ error for $\gamma \leq \frac{1}{4}$ ). 
ML hereafter) decoding, and those based on iterative decoding techniques. Note that by simply guessing the noise weight, the problem reduces to a permuted kernel problem [25].

Information Set Decoding. consists in picking $d_{P}$ bits at random among the $\ell$ output bits and perform a Gaussian elimination on the corresponding columns of the generator matrix of the LFSR. Decoding succeeds if there are no error among the selected bits, namely with probability $\left(\frac{1}{2}+\frac{\gamma}{2}\right)^{d_{P}}$. By iterating this simple algorithm enough time to get the correct decoding we can decode within a complexity roughly $\left(\frac{1}{2}+\frac{\gamma}{2}\right)^{-d_{P}}$ (improved decoding algorithms like 3 . make it possible to neglect the cost of the Gaussian elimination). When the bias $\gamma$ is too small, namely for $\gamma \leq 2^{1-c_{\text {hard }} / d_{P}}-1$, this requires over $2^{c_{\text {hard }}}$ iterations.

The converse approach consisting in trying to guess the error bits could seem interesting when the level of noise is low ( $\gamma$ close to 1$)$, but it is never more efficient than Information Set Decoding.

Maximum Likelihood Decoding. consists in trying to find the most likely $X$, given the $\ell$-bit output stream we have. As the source $\mathcal{S}_{\gamma}$ is memoryless with a positive bias, the maximum likelihood corresponds to the $X$ generating the closest (in terms of Hamming distance) codeword to $Y$.

This gives us a basic ML decoding algorithm: try all the possible initializations for $\mathcal{L}_{P}$ and sort them according to their distance to $Y$. This is however very costly (about $\mathcal{O}\left(2^{d_{P}} \ell\right)$ ) and can be slightly improved to $\mathcal{O}\left(2^{d_{P}} d_{P}\right)$ by using a Walsh transform as done in [19]. This method is successful if the solution is on top of the list. This is the case only if we try to transmit less data than the channel capacity. For $d_{P} \leq c_{\text {easy }}$ and $\frac{1}{4} \geq \gamma \geq \sqrt{\frac{d_{P}}{\ell} 2 \log 2}$ (the $\frac{1}{4}$ is here to ensure that the approximation is valid), we can thus efficiently solve the problem. On the contrary, decoding can be impossible for two different reasons: either the noise is too high (that is, the bias is too small) and we sent more data than the channel capacity (for instance, if $\gamma \leq 1.18 \ell^{-\frac{1}{2}}$, the channel cannot transmit more than one bit of information, which means $Y$ cannot contain more than one bit of information on $X$ ), or the cost of the maximum likelihood decoding algorithm is too high.

First case - If we send more data than the capacity of the channel we will not be able to recover $X$ completely, but we can still get some information on it. This would not be sufficient to solve a decoding problem, but this is already enough to threaten a cryptographic construction.

The difference between the distance of $Y$ to any incorrect codeword and the distance to the correct one can be approximated to a normal law of expected value $-\gamma \frac{\ell}{2}$ and variance $\frac{\ell}{2}$ so the expected rank of the correct $X$ in the maximum likelihood list is approximately $1+\left(2^{d_{P}}-1\right) \varphi\left(\frac{-\gamma}{2} \sqrt{\ell}\right)$ where

$$
\varphi(x)=\frac{1}{\sqrt{2 \pi}} \int_{-\infty}^{x} e^{-\frac{t^{2}}{2}} d t .
$$

The amount of information one can get on $X$ depends directly on this rank. 
Second case - If the complexity of the ML decoding algorithm is too high, we can try too improve it. More subtle algorithms exist to recover the initialization of $\mathcal{L}_{P}$ : they cannot decode as much noise as the basic algorithm but can have a significantly lower complexity. The basic idea is that instead of decoding in the full code it is possible to decode only in a subcode and then extend the decoding to the rest of the code. For example, one could write the generator matrix of the code defined by $P$ and only consider columns ending with $d_{P}-k$ zeros: this way one would have to decode in a code of dimension $k$ (instead of $d_{P}$ ) but with less bits, that is a length $\ell .2^{-\left(d_{P}-k\right)}$ on average. When $\gamma$ is small, the right $X$ tops the ML list if $\frac{k}{\ell .2^{-\left(d_{P}-k\right)}} \leq \frac{\gamma^{2}}{2 \log 2}$. In general, it takes time $2^{k} \varphi\left(-\frac{\gamma}{2} \sqrt{\ell 2^{-\left(d_{P}-k\right)}}\right)$ to find the right $k$ bits of $X$.

This improvement makes it possible to recover the initialization of an LFSR at a lower cost, provided enough output bits are available. This approach can still be improved: if too few output bits are available, we can compute new ones from those we have. If we write all the output bits which are the XOR of $d$ different bits, starting from $\ell$ bits we can obtain $\left(\begin{array}{l}\ell \\ d\end{array}\right) \simeq \frac{\ell^{d}}{d !}$ bits instead of $\ell$, but with a noise of bias $\gamma^{d}$ instead of $\gamma$. This means that, for any value of $d$ (the size of the combinations we consider) and $k$ (the dimension of the subcode we obtain) we can decode if:

$$
\frac{k d !}{\ell^{d} \cdot 2^{-\left(d_{P}-k\right)}} \leq \frac{\gamma^{2 d}}{2 \log 2}
$$

In general, even if the previous bound is not reached, the correct $\mathrm{X}$ has rank:

$$
2^{k} \varphi\left(-\frac{\gamma^{d}}{2} \sqrt{\frac{\ell^{d}}{d !} 2^{-\left(d_{P}-k\right)}}\right) .
$$

The complexity of the decoding is then the sum of the three following steps:

- Computing the combinations: $\mathcal{O}\left(\frac{\ell^{d}}{d !} \times k\right)$

- Decoding (with a Walsh Transform): $\mathcal{O}\left(k 2^{k}\right)$.

- Finding the right codeword in the ML list: $\mathcal{O}\left(2^{k} \varphi\left(-\frac{\gamma^{d}}{2} \sqrt{\frac{\ell^{d}}{d !} 2^{-\left(d_{P}-k\right)}}\right)\right)$

If one cannot pay more than $\mathcal{O}\left(c_{\text {hard }} 2^{c_{\text {hard }}}\right)$ complexity, we must have $k \leq c_{\text {hard }}$ and $d$ roughly less than $c_{\text {hard }} \frac{\log 2}{\log \ell}$ so that $\frac{\ell^{d}}{d !} \leq 2^{c_{\text {hard }}}$. The rank in the ML list is $2^{k} \varphi(-t)$ where $t \leq \frac{\gamma^{d}}{2} 2^{c_{\text {hard }}-\frac{d_{P}}{2}}$. For $d_{P} \geq 2 c_{\text {hard }}$ we have $t \leq \frac{1}{2}$. So the rank is higher than $2^{k} \varphi\left(-\frac{1}{2}\right) \geq 0.3 \cdot 2^{k}$. So, this algorithm yields less than one bit of information of $X$.

Iterative Decoding. techniques are less efficient in terms of error correction, but can be applied to longer LFSRs. The idea is to find low-weight multiples of $P$ which form some parity check equations, and use them to decode as in a Low Density Parity Check (LDPC) code. 
As stated in [4, iterative decoding using parity check equations of weight $d \geq 4$ (that is multiples of $P$ of weight $d$ ), succeeds if it is possible to find enough of these parity check equations for the iterative process to converge. Decoding is thus possible if:

$$
\ell \geq 2^{\alpha_{d}(\gamma)+\frac{d_{P}}{d-1}} \quad \text { with } \quad \alpha_{d}(\gamma)=\frac{1}{d-1} \log _{2}\left[(d-1) ! \frac{1}{C\left(\gamma^{d-2}\right)}\right]
$$

From this, we can see that whatever the parameters of the system, there exists a $d$ which makes iterative decoding possible. This means that for the smallest $d$ satisfying this equation, there should be about just enough parity check equations: finding them requires to find nearly all multiples of $P$ of weight $d$ and degree less than $\ell$. Among the techniques described in Section 2.1, the Strategy 1 based on the birthday paradox is the most efficient. It has a cost:

$$
\mathcal{C}_{\text {parity }}=\left(\begin{array}{c}
\ell-1 \\
\left\lceil\frac{d-1}{2}\right\rceil
\end{array}\right) \Leftrightarrow \log _{2}\left(\mathcal{C}_{\text {parity }}\right) \simeq \frac{d-1}{2} \log _{2} \ell-\log _{2}\left(\frac{d-1}{2} !\right) .
$$

The cost for the decoding is then negligible compared to this cost. This means that if one cannot pay more than $2^{\text {chard }}$ to decode, one can only decode if $2^{c_{\text {hard }}} \geq$ $\mathcal{C}_{\text {parity }}$. Mixing Equations (4) and (5) we see that one can decode only if:

$$
c_{\text {hard }} \geq \frac{1}{2}\left[\log _{2}(d-1) !-\log _{2} C\left(\gamma^{d-2}\right)+d_{P}\right]-\log _{2}\left(\frac{d-1}{2} !\right) .
$$

Roughly, we get the same constraint: if $d_{P} \geq 2 c_{\text {hard }}$, decoding is not possible.

Property 4 (Noisy LFSR Decoding). The noisy LFSR decoding problem can efficiently be solved when $d_{P} \leq c_{\text {easy }}$ and $\frac{1}{4} \geq \gamma \geq \sqrt{\frac{d_{P} 2 \log 2}{\ell}}$. For $\gamma \leq$ $1.18 \ell^{-\frac{1}{2}}, Y$ contains less than 1 bit of information of $X$, that is, the mutual information between $X$ and $Y$ is smaller than 1 .

Assumption 5 (Noisy LFSR Decoding). The noisy LFSR decoding is hard on average when $P$ is generated by $\mathrm{Gen}_{1}$ (as specified in Problem 1) and when $d_{P} \geq 2 c_{\text {hard }}$ and $\gamma \leq 2^{1-c_{\text {hard }} / d_{P}}-1$. When $d_{\text {min }} \geq 2 c_{\text {hard }}, \gamma \leq 2^{1-c_{\text {hard }} / d_{\text {min }}}-1, P$ is generated by $\mathrm{Gen}_{2}$, and $w$ and $d_{K}$ are such that the Low-Weight Polynomial Multiple is hard, the noisy LFSR decoding is hard as well.

\subsection{The Hidden Correlation Problem}

We combine the previous problems into Hidden Correlation (HC) problem.

Problem 6 (Hidden Correlation).

Parameters: a length $\ell$, two relatively prime 2 polynomials $P$ and $Q$ of degree $d_{P}$ and $d_{Q}$ respectively, a bias $\gamma$

\footnotetext{
${ }^{2}$ When $P$ and $Q$ have a common factor, the decoding problem is ambiguous so we exclude those cases. As we will see later, we will always choose distinct primitive polynomials $P$ and $Q$ so they never have a common factor.
} 
HC channel: given a uniformly distributed random seed $X$ of length $d_{Q}$, generate $Y$, the XOR of the output of length $\ell$ of $\mathcal{L}_{Q}$ initialized with $X$, the output of $\mathcal{L}_{P}$ initialized at random, and a random noise generated by $\mathcal{S}_{\gamma}$ (both random sources being independent)

Problem: given $Y$, recover $X$

As for the noisy LFSR decoding problem, we say that the problem is hard if no algorithm that outputs the correct $X$ with probability higher than $2^{1-c_{\text {hard }}}$ and average complexity less than $2^{\text {chard }}$ exists.

This problem is meant to be used given an oracle that solves the noisy LFSR decoding problem with parameters $\left(\ell^{\prime}, Q, \gamma^{\prime}\right)$ where $\ell^{\prime}=\ell-d_{K}$ for some degree $d_{K}$ and $\gamma^{\prime}=\gamma^{w}$ for some weight $w$. There are three main strategies to solve it:

1. consider $\mathcal{L}_{P} \oplus \mathcal{L}_{Q}$ as a single LFSR $\mathcal{L}_{P \times Q}$, recover its initializations and deduce the initializations of both $\mathcal{L}_{P}$ and $\mathcal{L}_{Q}$ from it, thus recovering $X$. Achieving this requires to be able to solve the noisy LFSR decoding problem with parameters $(\ell, P \times Q, \gamma)$.

2. suppress the output of $\mathcal{L}_{P}$ in $Y$ and decode $X$ with a shorter output and a higher noise level. One should first find a polynomial $K$, multiple of $P$ of degree $d_{K}$ and weight $w$ (note that choosing $K=P$ is also possible). Then multiply $Y$ by $K$ to suppress the influence of $\mathcal{L}_{P}$ and try to solve the noisy LFSR decoding problem with parameters $\left(\ell-d_{K}, Q, \gamma^{w}\right)$.

As discussed in Section 2.1 the problem of finding $K$ can be hard. The key idea of our construction is that this $K$ can be a trapdoor.

3. suppress the output of $\mathcal{L}_{Q}$ in $Y$ and recover the initialization of $\mathcal{L}_{P}$ (with a shorter output and a higher noise level). Once this is done, recovering $X$ consists in decoding in $\mathcal{L}_{Q} \oplus \mathcal{S}_{\gamma}$ only, with the full output $\ell$ and the same bias $\gamma$ : the oracle can do this. We can use the same method as above and find a multiple $K$ of $Q$. In the end, we need to solve the noisy LFSR problem with parameters $\left(\ell-d_{K}, P, \gamma^{w}\right)$.

By taking $P$ random of degree $d_{P} \geq 2 c_{\text {hard }}$ and $\gamma \leq 2^{1-c_{\text {hard }}} / d_{P}-1$ we know from Assumption 5 that the decoding problem for $\mathcal{L}_{P} \oplus \mathcal{S}_{\gamma}$ is intractable. This renders strategies 1 and 3 impossible.

To build a public-key cryptosystem on this problem, we need to find parameters which render strategy 2 computationally infeasible, and at the same time let us have a trapdoor polynomial $K$ making it possible to solve it. We do this by proving an upper bound on the information one can get on $X$ using strategy 2 in the favorable case where we can compute all multiples of low weight (except the hidden one), and bounded by the maximum number of iterations $\left(2^{\text {chard }}\right)$ we can manage. The number of possibles multiples of weight $w$ and degree $d_{K} \leq \ell$ (except the hidden $K$ ) is given by equation (11).

For each multiple $K^{\prime}$ of $P$ of weight $i$ it is possible to suppress the influence of $\mathcal{L}_{P}$ and recover a little bit of information on $X$. This information $I_{i}$ is bounded by $I_{i} \leq \ell C\left(\gamma^{i}\right)$.

By ignoring the cost of finding a multiple of weight $i$ we upper bound the cost of recovering the $I_{i}$ bits of information by $\mathcal{O}(\ell i)$, the cost of computing 
$Y^{\prime}=Y \cdot K^{\prime}$. Then an adversary can use all possible multiples of a given weight, as long as there are less than $\frac{1}{\ell i} 2^{c \text { hard }}$ such multiples. For large values of $i$, when there are more multiples of $P$, he is limited to exactly $\frac{1}{\ell i} 2^{c_{\text {hard }}}$ multiples. The total information one can get is at most:

$$
\mathcal{I}=\sum_{i=2}^{\infty} \ell C\left(\gamma^{i}\right) \min \left(\frac{\left(\begin{array}{l}
\ell \\
i
\end{array}\right)}{2^{d_{P}}}, \frac{2^{c_{\text {hard }}}}{\ell i}\right) .
$$

We can consider that this attack is not a threat if $\mathcal{I} \leq 1$ (which means that after using about $2^{\text {chard }}$ polynomials, an adversary gets less than 1 bit of information on $X)$. This bound is not tight since we neglected the cost of finding the multiples.

Assumption 7 (Hidden Correlation Problem). When $d_{P} \geq 2 c_{\text {hard }}, \gamma \leq$ $2^{1-c_{\text {hard }} / d_{P}}-1$, and $\mathcal{I} \leq 1$, the Hidden Correlation problem is hard on average when $P$ is generated by $\mathrm{Gen}_{1}$ as specified in Problem 1. When $d_{\min } \geq 2 c_{\text {hard }}$, $\gamma \leq 2^{1-c_{\text {hard }} / d_{\min }}-1, \mathcal{I} \leq 1$ (with $d_{P}$ replaced by $d_{\min }$ ), $P$ is generated by $\mathrm{Gen}_{2}$, and $w$ and $d_{K}$ are such that the Low-Weight Polynomial Multiple is hard, the hidden correlation problem is hard as well.

\section{TCHo Encryption}

\subsection{Specifications}

This construction depends on a number of domain parameters. These are:

- a bias $\gamma \in[0,1]$ (we only consider positive biases here),

- two integers $d_{\min }$ and $d_{\max }$ bounding the degree of $P: d_{P} \in\left[d_{\min }, d_{\max }\right]$.

- two integers $d_{Q}$ and $d_{K}$ : the degrees of polynomials $Q$ and $K$,

- a primitive polynomial $Q$ of degree $d_{Q}$

- an integer $w$ corresponding to the weight of the polynomial $K$,

- an integer $\ell$ which is the length of the produced stream.

As we will see later in Table 1, suitable parameters might look like:

$$
\gamma=0.98, d_{P} \in[6000,6600], d_{Q}=20, d_{K}=11560, w=99, \ell=13080 .
$$

Key generation. The public key is a primitive polynomial $P$. The private key is a low-weight multiple $K$ of $P$.

To generate such a key pair one will first pick a random binary polynomial $K$ coprime with $Q$ of given weight $w$ and degree $d_{K}$. Then one factors this polynomial and checks if it has a primitive factor of suitable degree $d_{P} \in\left[d_{\min }, d_{\max }\right]$. If such a factor exists one uses it as the public key $P$ and a key pair was found, otherwise one picks another random polynomial $K$, factors it, etc. The complexity of this step is detailed in Appendix $\mathrm{A}$. With the above parameters, this takes a little more than 4 minutes using NTL [27] on a $1.5 \mathrm{GHz}$ Pentium 4. 


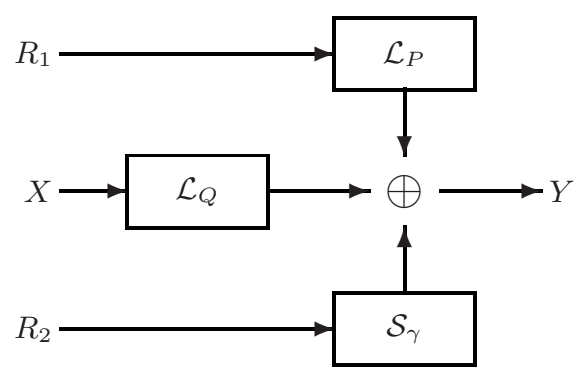

Fig. 1. Encryption

Encryption. The core of the system is a stream-cipher-like construction, built as the XOR of two LFSRs $\mathcal{L}_{P}$ and $\mathcal{L}_{Q}$ (with respective retroaction polynomials $P$ and $Q$ ) and a biased random source $\mathcal{S}_{\gamma}$ where $0 \leq \gamma \leq 1$ represents the bias of this source.

The word $X$ of $d_{Q}$ bits to be encrypted is used to initialize $\mathcal{L}_{Q} \cdot \mathcal{L}_{P}$ is randomly initialized with a string $R_{1}$. $R_{2}$ denotes the random coins for $\mathcal{S}_{\gamma}$. We assume that $R_{1}$ and $R_{2}$ are independent. The ciphertext is the binary stream $Y$ of length $\ell$ equal to the XOR of the streams generated by $\mathcal{L}_{P}, \mathcal{L}_{Q}$ and $\mathcal{S}_{\gamma}$ (see Fig. 1). We denote $Y=\operatorname{TCHo}_{P}(X ; R)$ for $R=\left(R_{1}, R_{2}\right)$. The cost of an encryption is $\mathcal{O}\left(\left(d_{P}+d_{Q}+\varepsilon_{\gamma}\right) \times \ell\right)$, where $\varepsilon_{\gamma}$ represents the cost of generating one random bit with bias $\gamma$. A dedicated hardware of $\mathcal{O}\left(d_{P}+d_{Q}+\varepsilon_{\gamma}\right)$ gates (typically, a few thousand gates) runs in time $\mathcal{O}(\ell)$ (typically, a few thousand clock cycles).

Decryption. To decrypt, one first uses the private key $K$ to suppress the influence of $\mathcal{L}_{P}$. For $i=1, \ldots, \ell-d_{K}$, compute $Y_{i}^{\prime}=\left(Y_{i}, Y_{i+1}, \ldots, Y_{i+d_{K}}\right) \bullet K$. This results in a new cipher $Y^{\prime}$ of length $\ell-d_{K}$ equal to the XOR of a stream generated by $\mathcal{L}_{Q}$ and a source of bias $\gamma^{w}$. The complexity is $\mathcal{O}\left(d_{K} \cdot \ell\right)$.

From Property 4 we know that if $\ell-d_{K} \geq \frac{2 d_{Q} \log 2}{\gamma^{2 w}}$ (and $\gamma^{w} \leq \frac{1}{4}$ ) we can recover the initialization of $\mathcal{L}_{Q}$ (thus $X$ ) using an ML decoding algorithm. Using a Walsh transform, this decryption costs $\mathcal{O}\left(d_{Q} 2^{d_{Q}}\right)$ operations. Finally, $X$ is recovered by solving a linear equation in time $\mathcal{O}\left(d_{K}^{3}\right)$. Overall decryption complexity is thus $\mathcal{O}\left(d_{K} \ell+d_{Q} 2^{d_{Q}}+d_{K}^{3}\right)$. Decoding is feasible for $d_{Q} \leq$ $c_{\text {easy }}$. However, that decryption is non-deterministic: there is a probability that $S_{\gamma}$ generates too much noise and that decryption returns an incorrect result. Bounds on this failure rate are hard to estimate as the linear code corresponding to a truncated LFSR is not as easy to study as for a full length LFSR. However, during all our tests, no decodings ever failed or returned the wrong plaintext. The bound used in Section 3.2 for parameter selection, taken from information theory, seems to be sufficient to have a negligible decryption failure rate. 
Table 1. Some suitable parameters for TCHo

\begin{tabular}{|c|c|c|c|c|c|c|c|c|}
\hline$c_{\text {hard }}$ & $d_{Q}$ & $d_{P}$ & $\gamma$ & $w$ & $d_{K}$ & $\ell$ & $\mathcal{I}$ & key gen. cost $^{3}$ \\
\hline \multirow[t]{7}{*}{80} & 20 & $500-600$ & 0.4 & 6 & 6200000 & 8000000 & $2^{-1.7}$ & $\bar{~} \sim 2^{56.5}$ \\
\hline & & $1000-1100$ & 0.74 & 13 & 78350 & 148000 & $2^{-1.9}$ & $\sim 2^{42.5}$ \\
\hline & & $3000-3500$ & 0.93 & 35 & 17100 & 21600 & $2^{-17}$ & $\sim 2^{36.5}$ \\
\hline & & $6000-6600$ & 0.98 & 99 & 11560 & 13080 & $2^{-1.1}$ & $\sim 2^{36}$ \\
\hline & & $6000-6060$ & 0.98 & 99 & 10620 & 12140 & $2^{-4}$ & $\sim 2^{39}$ \\
\hline & 30 & $1000-1500$ & 0.7 & 11 & 232000 & 339000 & $2^{-7}$ & $\sim 2^{44.5}$ \\
\hline & & $6000-6600$ & 0.979 & 93 & 12150 & 14150 & $2^{-2.1}$ & $\sim 2^{36}$ \\
\hline \multirow[t]{2}{*}{90} & 20 & $7000-7700$ & 0.98 & 105 & 13950 & 15900 & $2^{-2.1}$ & $\sim 2^{36.5}$ \\
\hline & 30 & $7000-7700$ & 0.98 & 99 & 14460 & 16750 & $2^{-0.1}$ & $\sim 2^{36.5}$ \\
\hline \multirow[t]{2}{*}{128} & 20 & $10000-11000$ & 0.977 & 108 & 25050 & 29300 & $2^{-5.8}$ & $\sim 2^{38.5}$ \\
\hline & 30 & $10000-11000$ & 0.977 & 102 & 26300 & 31100 & $2^{-2.9}$ & $\sim 2^{38.5}$ \\
\hline
\end{tabular}

Encrypting larger blocks. To encrypt $n$ blocks $X_{1}, \ldots, X_{n}$ we define TCHo ${ }^{n}$, the concatenation of $n$ independent instances of TCHo (thus able to encrypt $n \cdot c_{\text {easy }}$ bits of data), by using independent random coins $R_{1}, \ldots, R_{n}$ :

$\operatorname{TCHo}_{P}^{n}\left(X_{1}, \ldots, X_{n} ; R_{1}, \ldots, R_{n}\right)=\left[Y_{1}, \ldots, Y_{n}\right]$, where $Y_{i}=\operatorname{TCHo}_{P}\left(X_{i} ; R_{i}\right)$.

\subsection{Parameters Selection}

We review here all the constraints on the system parameters in order for a legitimate decryption to be possible and for an attack by a computationally bounded adversary (bounded by $\mathcal{O}\left(2^{c_{\text {hard }}}\right)$ operations) to be impossible. As constraints need to be satisfied for any $d_{P} \in\left[d_{\min }, d_{\max }\right]$, in the following equations, $d_{P}$ was replaced either by $d_{\min }$ or by $d_{\max }$ depending on the type of inequality.

- legitimate decryption is possible if: $\ell-d_{K} \geq \frac{2 d_{Q} \log 2}{\gamma^{2 w}}, \gamma^{w} \leq \frac{1}{4}$, and $d_{Q} \leq c_{\text {easy }}$ (see Section 2.3).

- recovering $K$ is impossible if the conditions of Assumption 2 are verified, that is: $w \log _{2} \frac{d_{K}}{d_{\max }}>c_{\text {hard }}$ and $\left(\begin{array}{c}d_{K} \\ w-1\end{array}\right) \leq 2^{d_{\min }}$.

- without $K$, decryption is equivalent to solving an instance of the HC problem. From Assumption 7 we know this is impossible if:

$$
d_{\text {min }} \geq 2 c_{\text {hard }}, \gamma \leq 2^{1-\frac{c_{\text {hard }}}{d_{\min }}}-1 \text {, and } \mathcal{I}=\sum_{i=2}^{\infty} \ell C\left(\gamma^{i}\right) \min \left(\frac{\left(\begin{array}{l}
\ell \\
i
\end{array}\right)}{2^{d_{\min }}}, \frac{2^{c_{\text {hard }}}}{\ell i}\right) \leq 1 .
$$

We are looking for parameters which satisfy all the inequalities stated above. One should first choose $d_{Q} \leq c_{\text {easy }}$ and $d_{\text {min }} \geq 2 c_{\text {hard }}$, and deduce the largest possible $\gamma=2^{1-c_{\text {hard }} / d_{\text {min }}}-1$. Then find a $w$ for which $\ell=\left(\ell-d_{K}\right)+d_{K} \geq \frac{2 d_{Q} \log 2}{\gamma^{2 w}}+$ $d_{\max } 2^{\frac{c_{\text {hard }}}{w}}$ is small enough and deduce the minimum $d_{K}$ and $\ell$. Once parameters

${ }^{3}$ This is the cost of generating a key when using an algorithm based on linear algebra.

See Appendix $\mathrm{A}$ for details. 
are obtained one can just check whether $\mathcal{I} \leq 1$ or not and maybe reduce $\gamma$ accordingly. Table 1 gives a few sets of parameters satisfying these inequalities. As one can notice, no small value of $d_{P}$ appear in the tables. This is mainly because parameters which would otherwise be suitable for all the constraints always lead to a value of $\mathcal{I}$ too large. This however is not a problem as the optimal values (in terms of encryption/decryption complexity and transmission rate) seem to appear for larger values of $d_{P}$.

\subsection{Security}

Key Recovery. The key generation algorithm we use corresponds exactly to the $\mathrm{Gen}_{2}$ generator of Assumption [2. If the conditions of this assumption are respected, the key recovery problem is exactly the problem of solving a hard instance of the low-weight polynomial multiple problem. For suitable parameters, key recovery should thus be computationally impossible.

Message Recovery. requires to solve the hidden correlation problem. Solving this problem is hard in general, thus with suitable parameters the encryption can be seen as a one-way function. In order to use the Fujisaki-Okamoto construction we need to prove that this encryption is $(t, \varepsilon)$-secure in the sense of One-Way Encryption (OWE) and $\Gamma$-uniform (see Appendix $\mathrm{B}$ for definitions).

Theorem 8. Under Assumptions 2, 5, and 7. TCHo is $\left(2^{c_{\text {hard }}}, \frac{2}{2^{d} Q}\right)-O W E-C P A-$ secure.

Proof. We have chosen parameters such that, for any value of $P$ and $K$, an adversary spending less than $\mathcal{O}\left(2^{c_{\text {hard }}}\right)$ time always gets less than 1 bit of information on the plaintext $X$ using a chosen plaintext attack (CPA). Therefore, he always has a probability lower than $\frac{2}{2^{d} Q}$ of guessing the correct $X$.

Theorem 9. The TCHo encryption scheme is $\left(\frac{1}{2}+\frac{\gamma}{2}\right)^{\ell}$-uniform.

Proof. We need to upper bound the probability (on the random coins $R_{1}$ and $R_{2}$ introduced in Section 3.1) that a given plaintext is mapped to a given ciphertext. As we only consider positive biases, for a given initialization of $\mathcal{L}_{P}$ the most likely ciphertexts correspond to the random coins $R_{2}$ giving $\mathcal{S}_{\gamma}=0$. This happens with probability $\left(\frac{1}{2}+\frac{\gamma}{2}\right)^{\ell}$. When taking the average on the possible initializations of $\mathcal{L}_{P}$ (on all the possible random coins $R_{1}$ ) this probability can only decrease.

Semantic Security. Deciding whether $Y$ encrypts $X$ under $\mathrm{TCHo}_{P}$ or is random is equivalent to deciding whether a binary string is an output form a noisy LFSR channel with parameters $\ell, P, \gamma$ or is random. This may be a hard problem as well. This question may deserve further work.

Malleability. Obviously, from $Y=\operatorname{TCHo}_{P}\left(X ;\left(R_{1}, R_{2}\right)\right)$, an adversary can forge a new ciphertext $Y^{\prime}=\mathrm{TCHo}_{P}\left(X \oplus \delta ;\left(R_{1} \oplus \rho, R_{2}\right)\right)$ without even knowing $X, R_{1}$ or $R_{2}$. Hence, raw TCHo is clearly not OWE-CCA-secure, just like the raw RSA cryptosystem. 
Extension to $\mathrm{TCHo}^{n}$. Let $\mathrm{TCHo}_{P}^{n}\left(X_{1}, \ldots, X_{n} ; R_{1}, \ldots, R_{n}\right)=\left[Y_{1}, \ldots, Y_{n}\right]$. An adversary $A$ knows $Y_{1}, \ldots, Y_{n}$ and $P$ and wants to recover $X_{1}, \ldots, X_{n}$. We know that spending less than $2^{c_{\text {hard }}}$ time, $Y_{i}$ cannot give $A$ more than 1 bit of information on $X_{i}$. The other $Y_{j}$ cannot help as $A$ can generate as many couples $\left(X_{j}, Y_{j}\right)$ as he wants by himself (he knows $P$ and nothing else is required for encryption) and this will not give him any information on $X_{i}$. As the $X_{i}$ are independent, if $A$ tries to guess the values of $X_{1}, \ldots X_{n}$ simultaneously he will not have a better probability of success than if he tries to guess them one after the other: his probability of success will be less or equal to $\left(\frac{2}{2^{d} Q}\right)^{n}$. The concatenation $\mathrm{TCHo}^{n}$ is thus $\left(2^{c_{\text {hard }}}, \frac{2^{n}}{2^{d} Q^{n}}\right)$-OWE-CPA-secure.

Concerning $\Gamma$-uniformity, the results also extend well for $\mathrm{TCHo}^{n}$. For the concatenation we have:

$$
\Gamma\left(X_{1}, \ldots, X_{n}, Y_{1}, \ldots, Y_{n}\right)=\operatorname{Pr}\left[h \leftarrow_{R} \text { COINS }: \forall i, Y_{i}=\mathcal{E}_{p k}\left(X_{i} ; h\right)\right] .
$$

As before, the best probability will be obtained if $\mathcal{S}_{\gamma}=0$ for each of the $n$ independent encryptions. This happens with probability $\left(\frac{1}{2}+\frac{\gamma}{2}\right)^{\ell n}$ and thus $\mathrm{TCHo}^{n}$ is $\left(\frac{1}{2}+\frac{\gamma}{2}\right)^{\ell n}$-uniform. We deduce that we can build, in the random oracle model, an IND-CCA secure hybrid cryptosystem TCHo ${ }^{n}$-FO based on $\mathrm{TCHo}^{n}$ and an FGsecure symmetric encryption, by using the Fujisaki-Okamoto construction [12].

\section{Conclusion}

We presented the first public-key cryptosystem which resembles a stream cipher. Stream ciphers are usually very efficient when it comes to low-cost hardware implementation, so implementation may be quite competitive on these platforms. Software implementations are pretty fast as well. One issue with our construction might be the need for a huge amount of random coins, but hardware entropy accumulators may supply them efficiently. The main drawback is currently the overhead size and the decryption complexity. Although quite reasonable, future work will decrease it. One option would consist in replacing $\mathcal{L}_{Q}$ by a better binary code. As suggested by Willi Meier, repetition codes seem to offer a very good decryption complexity and can be available for higher dimensions.

TCHo is currently a prototype. Our concept might still have to be improved before becoming a real product, but TCHo is definitely a first step in a new direction for public-key cryptography. We encourage analysis of TCHo since it will either demonstrate its security or lead to improvements on stream cipher attacks.

\section{References}

1. Avoine, G., Oechslin, P.: A scalable and provably secure hash-based RFID protocol. In: PerSec 2005 (2005)

2. Camion, P., Mihaljević, M.J., Imai, H.: Two alerts for design of certain stream ciphers: Trapped LFSR and weak resilient function over GF(q). In: Nyberg, K., Heys, H.M. (eds.) SAC 2002. LNCS, vol. 2595, pp. 196-213. Springer, Heidelberg (2003) 
3. Canteaut, A., Chabaud, F.: A new algorithm for finding minimum-weight words in a linear code: Application to McEliece's cryptosystem and to narrow-sense BCH codes of length 511. IEEE Transactions on Information Theory 44(1), 367-378 (1998)

4. Canteaut, A., Trabbia, M.: Improved fast correlation attacks using parity check equations of weight 4 and 5. In: Preneel, B. (ed.) EUROCRYPT 2000. LNCS, vol. 1807, pp. 573-588. Springer, Heidelberg (2000)

5. Chepyshov, V.V., Johansson, T., Smeets, B.: A simple algorithm for fast correlation attacks on stream ciphers. In: Schneier, B. (ed.) FSE 2000. LNCS, vol. 1978, pp. 181-195. Springer, Heidelberg (2001)

6. Courtois, N., Klimov, A., Patarin, J., Shamir, A.: Efficient algorithms for solving overdefined systems of multivariate polynomial equations. In: Preneel, B. (ed.) EUROCRYPT 2000. LNCS, vol. 1807, pp. 392-407. Springer, Heidelberg (2000)

7. Courtois, N., Meier, W.: Algebraic attacks on stream ciphers with linear feedback. In: Biham, E. (ed.) Advances in Cryptology - EUROCRPYT 2003. LNCS, vol. 2656, pp. 345-359. Springer, Heidelberg (2003)

8. Courtois, N., Pieprzyk, J.: Cryptanalysis of block ciphers with overdefined systems of equations. In: Zheng, Y. (ed.) ASIACRYPT 2002. LNCS, vol. 2501, pp. 267-287. Springer, Heidelberg (2002)

9. Dalai, D.K., Gupta, K.C., Maitra, S.: Results on algebraic immunity for cryptographically significant boolean functions. In: Canteaut, A., Viswanathan, K. (eds.) INDOCRYPT 2004. LNCS, vol. 3348, pp. 92-106. Springer, Heidelberg (2004)

10. Faugère, J.-C.: A new efficient algorithm for computing gröbner bases (F4). Journal of Pure and Applied Algebra 139, 61-88 (1999)

11. Faugère, J.-C.: A new efficient algorithm for computing gröbner bases without reduction to zero (F5). In: ISSAC 2002, Lille, France, July 2002, pp. 75-83. ACM, New York (2002)

12. Fujisaki, E., Okamoto, T.: Secure integration of asymmetric and symmetric encryption schemes. In: Wiener, M. (ed.) CRYPTO 1999. LNCS, vol. 1666, pp. 537-554. Springer, Heidelberg (1999)

13. Johansson, T., Jönsson, F.: Fast correlation attacks based on turbo code techniques. In: Wiener, M.J. (ed.) CRYPTO 1999. LNCS, vol. 1666, pp. 181-197. Springer, Heidelberg (1999)

14. Johansson, T., Jönsson, F.: Improved fast correlation attacks on stream ciphers via convolutional codes. In: Stern, J. (ed.) EUROCRYPT 1999. LNCS, vol. 1592, pp. 347-362. Springer, Heidelberg (1999)

15. Johansson, T., Jönsson, F.: Fast correlation attacks through reconstruction of linear polynomials. In: Bellare, M. (ed.) CRYPTO 2000. LNCS, vol. 1880, pp. 300-315. Springer, Heidelberg (2000)

16. Lee, P.J., Brickell, E.F.: An observation on the security of McEliece's public-key cryptosystem. In: Günther, C.G. (ed.) EUROCRYPT 1988. LNCS, vol. 330, pp. 275-280. Springer, Heidelberg (1988)

17. Lu, Y.: Applied Stream Ciphers in Mobile Communications. Phd thesis num. 3491, EPFL (2006), http://library.epfl.ch/theses/?nr=3491

18. Lu, Y., Meier, W., Vaudenay, S.: The conditional correlation attack: A practical attack on bluetooth encryption. In: Shoup, V. (ed.) CRYPTO 2005. LNCS, vol. 3621, pp. 97-117. Springer, Heidelberg (2005)

19. Lu, Y., Vaudenay, S.: Faster correlation attack on bluetooth keystream generator E0. In: Franklin, M. (ed.) CRYPTO 2004. LNCS, vol. 3152, pp. 407-425. Springer, Heidelberg (2004) 
20. Meier, W., Staffelbach, O.: Fast correltaion attacks on stream ciphers. In: Günther, C.G. (ed.) EUROCRYPT 1988. LNCS, vol. 330, pp. 301-314. Springer, Heidelberg (1988)

21. Mihaljevic, M.J., Fossorier, M.P.C., Imai, H.: A low-complexity and highperformance algorithm for the fast correlation attack. In: Schneier, B. (ed.) FSE 2000. LNCS, vol. 1978, pp. 196-212. Springer, Heidelberg (2001)

22. Molland, H., Mathiassen, J.E., Helleseth, T.: Improved fast correlation attack using low rate codes. In: Paterson, K.G. (ed.) Cryptography and Coding. LNCS, vol. 2898, pp. 67-81. Springer, Heidelberg (2003)

23. Molnar, D., Wagner, D.: Privacy and security in library RFID: issues, practices, and architectures. In: Atluri, V., Pfitzmann, B., McDaniel, P.D. (eds.) CCS 2004, pp. 210-219. ACM Press, New York (2004)

24. Rivest, R.L, Shamir, A., Adleman, L.M.: A method for obtaining digital signatures and public-key cryptosystems. Communications of the ACM 21(2), 120-126 (1978)

25. Shamir, A.: An efficient identification scheme based on permuted kernels (extended abstract). In: Brassard, G. (ed.) CRYPTO 1989. LNCS, vol. 435, pp. 606-609. Springer, Heidelberg (1990)

26. Shor, P.W.: Polynomial-time algorithms for prime factorization and discrete logarithms on a quantum computer. SIAM J. Comput. 26(5), 1484-1509 (1997)

27. Shoup, V.: NTL: A library for doing number theory. Available online from http://www.shoup.net/ntl/

28. Siegenthaler, T.: Cryptanalysts representation of nonlinearly filtered MLsequences. In: Pichler, F. (ed.) EUROCRYPT 1985. LNCS, vol. 219, pp. 103-110. Springer, Heidelberg (1986)

29. von zur Gathen, J., Gerhard, J.: Modern Computer Algebra, 2nd edn. Cambridge University Press, Cambridge (2003)

30. Wagner, D.: A generalized birthday problem. In: Yung, M. (ed.) CRYPTO 2002. LNCS, vol. 2442, pp. 288-304. Springer, Heidelberg (2002)

\section{A Key Generation}

Average number of trials. The cost of a key generation depends directly on the number of trials required before finding a suitable key pair. Here we try to evaluate the probability that a random low-weight binary polynomial has a primitive factor of degree $d_{P}$.

It is known that, asymptotically, the number of irreducible binary polynomials of degree $d$ is equivalent to $\frac{2^{d}}{d}$. If we call $P$ one of these irreducible polynomials, a random binary polynomial $K$ of degree $d_{K}>d$ is divisible by $P$ (and has $P$ in his factorization) if $K \equiv 0 \bmod P$. The probability this happens is $2^{-d}$. Taking into account this probability and the number of irreducible polynomials, we obtain the probability that $K$ has an irreducible factor of degree $d$ is approximately $\frac{1}{d}$. Given that $P$ is irreducible, the probability that it is primitive is the probability that the logarithm of $x$ in $\left(\mathbf{F}_{2}[x] / P(x)\right)^{*}$ is coprime with $2^{d}-1$. The probability that two random numbers are coprime relates to Buffon's needle problem and is $\frac{6}{\pi^{2}}$. Given that $2^{d}-1$ is odd for sure, the probability that $P$ is primitive given that it is irreducible can be heuristically approximated to $\frac{8}{\pi^{2}} \approx 81 \%$.

This is true asymptotically for random polynomials. In our case we only consider polynomials $K$ of low weight. For this particular case we have no proof 
that the same result still hold 4 . However, experimental statistics tend to prove that this approximation is still very accurate for low-weight polynomials. The average number of trials is thus approximately $\frac{\pi^{2}}{8} d_{P}$.

Factoring algorithm. Concerning the algorithm to be used for the factorization, the choice is very wide. The straightforward method would be to use a generic factoring algorithm like Berlekamp's or Cantor-Zassenhaus (see Chapter 14 of 29. for more details on polynomial factorization). However, when dealing with high degree polynomials, methods based on linear algebra tend to use a lot of memory and degrees above $2^{16}$ are too high for standard computers. Moreover, in our case, a complete factorization is not required: it is enough to check whether our polynomial has an irreducible factor of degree $d_{P}$, and this can be done quite efficiently.

The polynomial $X^{2^{d}}+X$ is the product of all binary irreducible polynomials whose degrees divide $d$. Checking if $K$ has a factor of degree $d_{P}$ reduces to the computation of $\operatorname{gcd}\left(X^{2^{d_{P}}}+X, K\right)$. If this gcd has a degree less than $d_{P}$ one can be sure that $K$ has no suitable factors, otherwise one simply needs to factor this "low degree" polynomial using whatever algorithm.

Computing this gcd is much faster than a complete factorization for high degree $K$ and a small $d_{P}$. The computation of $X^{2^{d} P}+X \bmod K$ using successive squarings can however be quite long when $d_{P}$ increases, thus, the best solution depends on the system parameters. Using Berlekamp's factoring algorithm the complexity of the factoring is $\mathcal{O}\left(d_{K}{ }^{2.4}\right.$ ) (where 2.4 is the cost for linear algebra) and using the gcd-based algorithm it is $\mathcal{O}\left(d_{P} d_{K}^{2}\right)$. Taking into account the number of iterations for a key generation we obtain $\mathcal{O}\left(d_{P} d_{K}^{2.4}\right)$ and $\mathcal{O}\left(d_{P}^{2} d_{K}^{2}\right)$. Cantor-Zassenhaus gives $\mathcal{O}\left(d_{P} d_{K}^{2} \log d_{K} \log \log d_{K}\right)$, which is the best complexity, but with a very large constant term.

Using degree ranges. The number of attempts required for the key generation to find a polynomial with degree $d_{P} \in\left[d_{\min }, d_{\max }\right]$ is divided by $\delta=$ $d_{\max }-d_{\min }+1$. If we use a general factoring algorithm, this directly divides the key generation complexity by $\delta$ but if we use the gcd method, the time used to compute $X^{2^{d} P}+X \bmod K$ does not change and we have to compute $\delta$ gcds. In both cases this improves the key generation complexity, but for large values of $\delta$, the gcd method becomes very slow. Using Berlekamp's algorithm the complexity becomes $\mathcal{O}\left(\frac{1}{\delta} d_{P} d_{K}^{2.4}\right)$, with Cantor-Zassenhaus it is $\mathcal{O}\left(\frac{1}{\delta} d_{P} d_{K}^{2} \log d_{K} \log \log d_{K}\right)$, and for the gcd-based technique it becomes $\mathcal{O}\left(\frac{d_{P}}{\delta}\left(d_{P}+\delta\right) d_{K}^{2}\right)$.

For small values of $\delta$ the best choice is the gcd-based technique, but for larger $\delta$ generic factoring algorithms are faster. If $d_{K}$ is large, Cantor-Zassenhaus is the best algorithm.

\footnotetext{
${ }^{4}$ In our construction we only considers polynomials $K$ with a non null constant term, which are therefore not divisible by $X$. If one chooses them with an odd weight they will never be divisible by $X+1$ and will never have an irreducible factor of degree 1. Some similar properties might also hold for other degrees!
} 
Primitivity testing. In general, checking whether an arbitrary polynomial is primitive is hard. However, we only need here to probabilistically filter out random polynomials which are not primitive. This is essentially easy. A probabilistic method for finding a generator of $\mathbf{Z}_{p}^{*}$ can be adapted to checking whether a polynomial is primitive. One simply need to find all divisors $p_{i}$ of $2^{d_{P}}-1$ smaller than a given $\lambda$, and check if the order of $X$ in $(G F(2)[X] / P(X))^{*} \operatorname{divides} \frac{2^{d} P-1}{p_{i}}$. If this is the case for none of the $p_{i}, P$ is primitive with probability $1-\lambda^{-1}$.

So, with complexity $\mathcal{O}\left(\mathcal{P}\right.$ oly $\left.\left(d_{P}\right) \sqrt{\lambda}\right)$ it is possible to reduce the risk that his algorithm accepts a non-primitive polynomial to $\mathcal{O}\left(\lambda^{-1}\right)$. It is thus possible to reach error rates as low as $2^{-40}$ with a cost negligible compared to the factorization step.

\section{B Definitions}

We report here definitions taken from the article by Fujisaki and Okamoto [12].

Definition 10 (Asymmetric Encryption). An asymmetric encryption scheme $\Pi$ is a triple of algorithms $\mathcal{K}, \mathcal{E}$ and $\mathcal{D}$, associated with two finite sets, $\operatorname{COINS}(k)$ (a set of random coins) and $\operatorname{MSPC}(k)$ (a message space), for $k \in \mathbb{N}$.

- $\mathcal{K}$, the key generation algorithm, is a probabilistic algorithm which on input $1^{k}(k \in \mathbb{N})$ outputs a pair of keys, $(p k, s k) \leftarrow \mathcal{K}\left(1^{k}\right)$.

$-\mathcal{E}$, the encryption algorithm, is a probabilistic algorithm that takes a key $p k$, an message $x \in \mathrm{MSPC}$ and a string $r \leftarrow_{R} \operatorname{COINS}(k)$, where $\leftarrow_{R}$ represents $a$ random affectation, and produces a ciphertext $y=\mathcal{E}_{p k}(x ; r)$.

- $\mathcal{D}$, the decryption algorithm, is a deterministic algorithm that takes a key sk and a ciphertext $y$ and returns a message $x \leftarrow \mathcal{D}_{s k}(y)$.

It is required that, for any $k \in \mathbb{N}$, if $(p k, s k) \leftarrow \mathcal{K}\left(1^{k}\right), x \in \operatorname{MSPC}$, and $y \leftarrow \mathcal{E}_{p k}(x)$, then $\mathcal{D}_{s k}(y)=x$.

Definition 11 (OWE). Let $\Pi=(\mathcal{K}, \mathcal{E}, \mathcal{D}$, COINS, MSPC) be an asymmetric encryption scheme. Let $A$ be an adversary knowing $y$ and $p k$ and trying to recover $x$. We say that $\Pi$ is $(t, \varepsilon)$-OWE-secure if, for any $A$ running in at most time $t$ and for any $x \in \mathrm{MSPC}$ :

$$
\operatorname{Pr}\left[(p k, s k) \leftarrow \mathcal{K}\left(1^{k}\right) ; y \leftarrow \mathcal{E}_{p k}(x): A(p k, y)=\mathcal{D}_{s k}(y)\right] \leq \varepsilon .
$$

In other words, the probability among all the possible key pairs for A of recovering $x$ from its encrypted value is bounded by $\varepsilon$.

Definition 12 ( $\Gamma$-uniformity). Let $\Pi=(\mathcal{K}, \mathcal{E}, \mathcal{D}$, COINS, MSPC) be an asymmetric encryption scheme. For given $(p k, s k) \leftarrow \mathcal{K}\left(1^{k}\right), x \in \operatorname{MSPC}$ and $y \in$ $\{0,1\}^{*}$, define

$$
\Gamma(x, y)=\operatorname{Pr}\left[h \leftarrow_{R} \text { COINS }: y=\mathcal{E}_{p k}(x ; h)\right] .
$$

We say that $\Pi$ is $\Gamma$-uniform, if, for any $(p k, s k) \leftarrow \mathcal{K}\left(1^{k}\right)$, any $x \in \mathrm{MSPC}$ and any $y \in\{0,1\}^{*}, \Gamma(x, y) \leq \Gamma$. 
Definition 13 (Find-Guess-security). Let $\Pi=(\mathcal{E}, \mathcal{D}, \mathrm{KSPC}, \mathrm{MSPC})$ be a symmetric encryption scheme where KSPC is the key space and MSPC is the message space. Let $A$ be an adversary. We say $\Pi$ is $(t, \varepsilon)-F G$-secure if, for any $A$ running in at most time $t$ :

$$
\begin{aligned}
\mid 2 \cdot \operatorname{Pr}[a \leftarrow R & \operatorname{KSPC} ;\left(x_{0}, x_{1}\right) \leftarrow A ; b \leftarrow_{R}\{0,1\} ; \\
y & \left.=\mathcal{E}_{a}\left(x_{b}\right): A\left(x_{0}, x_{1}, y\right)=b\right]-1 \mid \leq \varepsilon .
\end{aligned}
$$

This means that an adversary choosing $x_{0}$ and $x_{1}$ cannot decide which of them corresponds to a given ciphertext $y=\mathcal{E}_{a}\left(x_{b}\right)$ with probability more than $\frac{1}{2}+\frac{\varepsilon}{2}$.

Definition 14 (IND-CCA-security). Let $\Pi=(\mathcal{K}, \mathcal{E}, \mathcal{D}$, COINS, MSPC) be an asymmetric encryption scheme and let $A$ be an adversary having access to a decryption oracle $\mathcal{D}_{s k}$. We say that $\Pi$ is $\left(t, q_{D}, \varepsilon\right)$-IND-CCA-secure if for any $A$ running in at most time $t$ and asking at most $q_{D}$ queries to the decryption oracle:

$$
\begin{aligned}
\mid 2 \cdot \operatorname{Pr}\left[(p k, s k) \leftarrow \mathcal{K}\left(1^{k}\right) ;\left(x_{0}, x_{1}\right)\right. & \leftarrow A^{\mathcal{D}_{s k}} ; b \leftarrow{ }_{R}\{0,1\} ; \\
y & \left.=\mathcal{E}_{p k}\left(x_{b}\right): A^{\mathcal{D}_{s k}}\left(x_{0}, x_{1}, y\right)=b\right]-1 \mid \leq \varepsilon .
\end{aligned}
$$

This means that an adversary choosing $x_{0}$ and $x_{1}$ cannot decide which of them corresponds to a given ciphertext $y=\mathcal{E}_{p k}\left(x_{b}\right)$ with probability more than $\frac{1}{2}+\frac{\varepsilon}{2}$. In the random oracle model, $\mathcal{E}_{\text {sk }}$ might also use some random oracles for encryption. We then get a similar definition of IND-CCA security by bounding the number of queries to these random oracles. 